\title{
Psychopathologie und operationalisierte Diagnostik in der forensischen Psychiatrie
}

\author{
Paul Hoff
}

Eingegangen: 1. Juli 2015 / Angenommen: 31. August 2015 / Online publiziert: 5. Oktober 2015

(C) Springer-Verlag Berlin Heidelberg 2015

\begin{abstract}
Zusammenfassung Der vorliegende Beitrag geht den folgenden Fragen nach: Wird eine systematische psychiatrische Klassifikation und Diagnostik, die über die Erfassung individueller Symptomkonstellationen hinausgeht, überhaupt (noch) gebraucht? Sind psychiatrische Klassifikation und Diagnostik das Ergebnis objektiver Messungen oder subjektiver Eindrücke? Erlaubt eine psychiatrische Diagnose eo ipso Rückschlüsse in juristischem Kontext? Hierzu werden eingangs der historische und der konzeptuelle Hintergrund der operationalisierten Diagnostik erläutert. Deren Kernelemente - deskriptives Vorgehen, explizite Kriterien bzw. Kriterienverbindungen für jede Diagnose, Orientierung am Schweregrad, Mehrachsigkeit, Komorbiditätsprinzip, nominalistisches Verständnis von Diagnosen und ätiologische Neutralität - werden kritisch untersucht und auf ihre Umsetzung in den gängigen Diagnosemanualen geprüft. Im Ergebnis dieser Auseinandersetzung wird postuliert, dass psychiatrische Diagnostik unbeschadet der Bedeutung methodischer Fragen ihr eigentliches Ziel nicht verfehlen darf: die Etablierung zuverlässiger und gültiger diagnostischer Termini als Grundlage ärztlichen Handelns sowohl in der Therapie als auch in der forensischen Begutachtung.
\end{abstract}

Schlüsselwörter Klassifikation · Nosologie $\cdot$ Historie · Psychische Störungen · Begutachtung

Prof. Dr. med. Dr. phil. P. Hoff $(\bowtie)$

Klinik für Psychiatrie, Psychotherapie und Psychosomatik,

Psychiatrische Universitätsklinik Zürich,

Lenggstr. 31,

Postfach 1931, 8032 Zürich, Schweiz

E-Mail: paul.hoff@puk.zh.ch

\section{Psychopathology and operationalized diagnostics in forensic psychiatry}

\begin{abstract}
This article discusses the following questions: are systematic psychiatric classification and diagnostics, which extend beyond the collation of individual symptom constellations, (still) needed at all? Are psychiatric classification and diagnostics the result of objective measurements or subjective impressions? Does a psychiatric diagnosis eo ipso allow conclusions in the juridical context? In the beginning the historical and conceptual background of operationalized diagnostics are elucidated. Their key elements - descriptive approach, explicit criteria and criteria associations for each diagnosis, orientation to the degree of severity, multiaxiality, comorbidity principle, nominalistic understanding of diagnoses and etiological neutrality - are critically investigated and tested for implementation in the commonly used diagnostic manuals. The results of this critical review confirm that psychiatric diagnostics must not fail to achieve the actual target irrespective of the significance of methodological questions, i.e. the establishment of reliable and valid diagnostic endpoints as the foundation of medical actions in the treatment as well as in forensic expert opinions.
\end{abstract}

Keywords Classification · Nosology $\cdot$ History $\cdot$ Mental disorders $\cdot$ Expert opinion

Psychopathologische Kenntnisse und die praktische Kompetenz, auf der Grundlage aller zu einer psychisch erkrankten Person vorliegenden Informationen eine reliable und valide Diagnose zu stellen, werden zu Recht als wesentliche Voraussetzungen psychiatrischen Handelns verstanden. Dies gilt für die ambulante und klinische, aber auch für die 
forensisch-psychiatrische Situation. Der technische Aspekt der Befunderhebung und Diagnostik, quasi die Messmethodik, darf allerdings nicht zuungunsten der inhaltlichen Frage, was denn eigentlich beschrieben und diagnostiziert werde, überbewertet werden. Im Hintergrund steht die Debatte um die Bedeutung von Reliabilität und Validität medizinischer Diagnosen: Dass es ohne reliable Datenerfassung in der empirischen Forschung keine validen Aussagen allgemeinerer Art, etwa zu Krankheitsentitäten oder Syndromen, geben kann, ist unbestritten. Weniger im Fokus steht oft die Tatsache, dass auch die unkritische Überbetonung der Reliabilität zu unerwünschten Verzerrungen führen kann.

Bei der nun schon über mehrere Jahrzehnte laufenden Diskussion über die Bedeutung der operationalisierten Diagnosemanuale in der Psychiatrie spielen diese Fragen eine große Rolle: Was wird durch ICD-10 $10^{1}$ und DSM- $5^{2}$-Diagnosen mit ihrer im Vergleich zu früheren diagnostischen Ansätzen klar verbesserten Reliabilität erfasst? Sind es Störungen, Syndrome, Krankheitsbilder oder „echte“ Krankheiten, also „natürliche Krankheitseinheiten“ im Sinne Emil Kraepelins bzw. „natural kinds“, wie es in der englischsprachigen Literatur heißt $[6,11,23,44,45]$ ?

Psychiatrische Diagnostik - so der konzeptuelle Rahmen der vorliegenden Arbeit - darf unbeschadet der Bedeutung methodischer Fragen ihr eigentliches Ziel nicht verfehlen, und dies ist die Etablierung zuverlässiger und gültiger diagnostischer Termini als Grundlage ärztlichen Handelns. Anders gewendet: Jede Beschäftigung mit der psychiatrischen Diagnostik, sei es in der Behandlung oder in der Begutachtung, verweist auf grundsätzliche Momente unseres Faches, die nicht ohne Verflachung des Diskurses ignoriert werden können.

Diagnostische Begriffe sind notwendig verknüpft mit nosologischen Modellen und deren theoretischen Fundamenten. Schon ein kurzer Blick auf die psychiatrische Ideengeschichte lehrt, dass so zentrale Termini wie Psychose, Neurose oder Persönlichkeitsstörung je nach theoretischer Matrix, in die sie eingebettet sind, Unterschiedliches meinen können, nicht ganz selten sogar Gegensätzliches. Der Begriff Neurose etwa adressierte in der ursprünglichen, von William Cullen (1710-1790) eingeführten Bedeutung eine nichtentzündliche Erkrankung des Nervensystems. Später bezog er sich auf psychopathologische Auffälligkeiten nichtpsychotischer Art, bei denen aber eine Zuordnung zu neuronalen Störungen gerade nicht gelang, wohl aber eine Verbindung zu psychodynamischen Vorgängen postuliert wurde. Die außerordentliche Heterogenität der Konzeptualisierung von „Neurose“ war zweifellos ein wesentlicher Grund dafür, dass die operationalen Diagnosemanuale ICD-

\footnotetext{
${ }^{1}$ Internationale statistische Klassifikation der Krankheiten und verwandter Gesundheitsprobleme, 10. Aufl.

${ }^{2}$ Diagnostic and Statistical Manual of Mental Disorders, 5. Aufl.
}

10 und DSM-5 auf seine Verwendung (nahezu) $)^{3}$ gänzlich verzichten.

Haben wir es im klinisch-therapeutischen Kontext mit einem anspruchsvollen Thema zu tun, so gilt dies genauso für die forensische Psychiatrie. Dort nämlich berührt die Diagnostik zwei Bereiche, die jenseits des engeren psychiatrischen Arbeitsfelds mit normativen Aspekten zu tun haben, also letztlich mit gesellschaftlichen Haltungen: Gemeint ist zum einen das Problem, wie im Fall eines strafrechtlich relevanten Verhaltens zu unterscheiden sei zwischen einer in bewusster Abwägung durchgeführten Regelüberschreitung, die zu bestrafen ist, und einer Regelüberschreitung, die als integraler Bestandteil einer gravierenden, das aktuelle Erleben und Verhalten der Person prägenden psychischen Störung zu verstehen ist. Letztere eröffnet die Optionen der Zuerkennung einer verminderten oder aufgehobenen Schuldfähigkeit. Popularisiert wird diese komplexe Thematik oft mit dem eingängigen Schlagwort: „bad or mad?“

Zum anderen geht es um die Abwägung zwischen zwei Grundwerten: Die personale Autonomie jedes einzelnen Menschen kann zu den Ansprüchen eines Individuums, aber auch der Gesellschaft auf persönliche und öffentliche Sicherheit in Widerspruch geraten. Die in der forensischen Psychiatrie tätige Fachperson bewegt sich in genau diesem schwierigen Umfeld. Sie muss, mindestens implizit, berücksichtigen, welches Ausmaß von Risiko eine Gesellschaft zu tragen bereit ist - und welches nicht. Der forensischen Psychiatrie kommt daher, nolens volens, eine maßgebliche Vermittlerrolle zwischen dem medizinischen und dem sozial-normativen Bereich zu. Dies rückt natürlich auch diagnostische Fragen in den Fokus des Interesses.

\section{Historischer und konzeptueller Hintergrund der operationalisierten Diagnostik}

Die Fragestellung, welcher Art und welchen Inhalts geeignete Kriterien für die Steuerung des diagnostischen Prozesses in der Psychiatrie zu sein haben, ist selbstverständlich nicht neu. Vielmehr begleitet sie das Fach seit seinen Anfängen als wissenschaftliche Disziplin, also mindestens seit der Epoche der Aufklärung im 18. Jh. Die meisten prägenden psychiatrischen Autoren haben sich intensiv mit ihr auseinandergesetzt. Vier von ihnen, die - aus unterschiedlichen Perspektiven - die Tradition der für ICD-10 und DSM-5 grundlegenden deskriptiven Psychopathologie geprägt haben, sollen skizzenhaft erwähnt werden: Emil Kraepelin, Karl Jaspers, Arthur Kronfeld und Kurt Schneider.

\footnotetext{
${ }^{3}$ In der ICD-10 wird noch die adjektivische Form „,neurotisch“ verwendet, nicht aber der Begriff „Neurose“ selbst. Im Stichwortverzeichnis des DSM-5 sucht der Leser „neurosis“/,neurotic“ vergeblich.
} 
Für Emil Kraepelin (1856-1926) als Vertreter einer stark am Krankheitsverlauf orientierten klinischen Forschung war die sorgfältige Erfassung des jeweiligen psychopathologischen Querschnittsbefunds von großer Bedeutung, um darauf eine aussagekräftige Langzeitbeurteilung aufbauen zu können. In seinem zwischen 1883 und 1927 in 9 Auflagen erschienenen einflussreichen Lehrbuch der Psychiatrie nahmen detailreiche Beschreibungen der klinischen Symptomatik einen kontinuierlich zunehmenden Raum ein. Hartnäckig betonte Kraepelin - als entschiedener, ja oft polemisch argumentierender Gegner der Psychoanalyse den streng deskriptiven Aspekt dieses Vorgangs: Psychische Zustände seien zu beobachten, im Idealfall sogar quantitativ $\mathrm{zu}$ vermessen und anschließend zu beschreiben, nicht aber zu deuten.

Karl Jaspers (1883-1969) verstand die Psychopathologie als, wie Janzarik [16] es später nennen sollte, Grundlagenwissenschaft der Psychiatrie. Sein Ziel war, sie auf eine solide und kontinuierlich reflektierte methodische Grundlage $\mathrm{zu}$ stellen und dadurch rein spekulativen und dogmatischen Ansätzen ihren Kredit zu entziehen. Nicht nur, aber auch in seinem psychiatrisch einflussreichsten Text Allgemeine Psychopathologie, erstmals 1913 erschienen [20], beschrieb er zum einen einzelne psychopathologische Phänomene mit großer klinischer Prägnanz, oft ergänzt durch Kasuistiken, zum anderen die Grundlagen der ungestörten Psyche. Sein hoher Differenzierungsgrad in der Beschreibung psychopathologischer Symptome hinderte Jaspers freilich nicht daran zu betonen, dass sich psychische Zustände niemals direkt, als solche, zeigen, sondern ,in Erscheinung treten“, im Dialog, im psychomotorischen Ausdruck und generell in der Interaktion zwischen zwei Personen. Er nannte dies die phänomenologische Forschungsrichtung in der Psychopathologie [19]. Dabei benutzte er den Begriff Phänomenologie recht eigenwillig, jedenfalls nicht im Sinne Hegels oder des späten Husserl, was später zu allerlei Missverständnissen Anlass gab (und gibt). Im jetzigen Kontext ist seine Grundhaltung von Interesse, die prägnant in der folgenden Formulierung zur Geltung kommt:

Wir müssen alle überkommenen Theorien, psychologischen Konstruktionen, alle bloßen Deutungen und Beurteilungen beiseite lassen, wir müssen uns rein dem zuwenden, was wir in seinem wirklichen Dasein verstehen, unterscheiden und beschreiben können ... Diese eigentümliche phänomenologische Vorurteilslosigkeit bei der Anschauung der Erscheinung als solcher ist nicht ursprünglicher Besitz, sondern mühsamer Erwerb nach kritischer Arbeit und oft vergeblichen Bemühungen. (Jaspers 1946, S. 48)

So sehr dies an den - später zu erläuternden - Anspruch der heutigen operationalen Diagnostik erinnert, möglichst wenige theoretische Vorannahmen in den diagnostischen
Prozess einfließen zu lassen, so sehr ist Jaspers' Votum eine eindringliche Warnung, die psychopathologische Befunderhebung nicht als einen einfachen und objektiven Datenerhebungsprozess misszuverstehen. Für ihn war eine gleichsam abschließende Erfassung der Ganzheit des psychisch gesunden oder gestörten Menschen in seiner biografischen Einzigartigkeit, seiner Personalität, letztlich mit keiner wissenschaftlichen Methode möglich.

Der heute weitgehend in Vergessenheit geratene Psychiater, Psychologe und Wissenschaftstheoretiker Arthur Kronfeld (1886-1941) stand zu seinem Heidelberger Kollegen Karl Jaspers in einem ebenso ideen- wie spannungsreichen Verhältnis. Manche Grundüberzeugung teilte er mit Jaspers, etwa diejenige der Irreduzibilität des Psychischen und des Sozialen als lebensweltlichen Erscheinungsformen der Conditio humana. Besonderen Wert legte er - v. a. in seinem Werk Das Wesen der psychiatrischen Erkenntnis von 1920 - auf die Verteidigung der methodischen Eigenständigkeit der Psychiatrie: Sie könne nur dann ,autologisch“ sein, wenn sie ihren „Forschungsgegenstand“, die erkrankte Person, zunächst mit eigenen, nämlich psychopathologischen Mitteln betrachte, und erst wenn diese an ihre Grenze gelangten, Methoden anderer Wissenschaftsfelder, „heterologische Methoden" in Kronfelds Worten, einsetze. Dies darf nicht im Sinne einer Wertung missverstanden werden: Ein heterologisches Vorgehen etwa neurowissenschaftlicher Art ist keineswegs per se schlechter, es ist lediglich anders als der primäre psychopathologische Zugang und soll ihn ergänzen, aber eben nicht ersetzen. Ganz im Sinne von Jaspers ging es Kronfeld um das kritische Reflektieren der Erkenntnisgrenzen der jeweiligen Methode, auch derjenigen der deskriptiven, von Jaspers ,phänomenologisch“ genannten Psychopathologie [7, 9, 22].

Ein wesentlicher Begründer der Heidelberger Psychiatrietradition war Kurt Schneider (1887-1967; [13, 17]). Methodenkritik und Selbstbeschränkung waren die Leitideen seines einflussreichsten Werks Klinische Psychopathologie, 2007 in 15. Auflage erschienen [36]. Schneider entwarf eine vorwiegend deskriptive, jedoch das Psychische gerade nicht atomisierende, sondern den klinisch sinnvollen, verstehenden Gesamtzusammenhang wahrende Psychopathologie. Kennzeichnend war dabei sein Ringen um eine präzise Begrifflichkeit. Dies führte etwa zur Herausarbeitung der „Symptome ersten Ranges“ in der Schizophreniediagnostik. Doch waren psychiatrische Diagnosen für ihn alles andere als die bloße Abbildung dessen, was Kraepelin unter „natürlichen Krankheitseinheiten“ verstanden hatte. Schneider betrachtete sie als vorläufige begriffliche Konstrukte, die sich der kontinuierlichen, durch empirisches Wissen und konzeptuelle Weiterentwicklung gesteuerten Adaptation zu stellen hätten. Ein solch nominaldefinitorische Auffassung psychiatrischer Diagnosen sowie die Forderung, möglichst eindeutige und schulenübergreifend 
akzeptierbare diagnostische Kriterien zu schaffen, machen Kurt Schneider zu einem entscheidenden Vorläufer der heutigen operationalisierten Diagnosemanuale.

Eine besondere Dynamik gewann die psychiatrische Diagnostikdebatte mit dem Aufkommen moderner Psychopharmaka ab den 60er Jahren des 20. Jh.s einerseits und den seither rasant wachsenden Möglichkeiten neurowissenschaftlicher Methoden andererseits (funktionale bildgebende Untersuchungen des Gehirns, neurophysiologische, neuropsychologische und molekulargenetische Methoden). All diese neuen, oft selbstbewusst auftretenden Bereiche waren (und sind) angewiesen auf eine reliable, replizierbare Erfassung möglichst homogener Patientengruppen, um in der Therapie- und Grundlagenforschung zu verallgemeinerbaren Erkenntnissen kommen zu können. Inkompatible diagnostische Modelle, die auf völlig divergenten, ja sich ausschließenden Vorannahmen beruhten, seien diese neurobiologischer, behavioristischer, hermeneutischer oder sozialwissenschaftlicher Provenienz, wurden zunehmend zum Problem, besser: zum Forschungshindernis [12, 31]. Gesucht war eine für forschende und klinische Psychiater jedweder theoretischer Ausrichtung akzeptable diagnostische Sprache, gleichsam ein größter gemeinsamer Nenner. Die Entwicklung der beiden heutigen Diagnosemanuale ICD-10 und DSM-5 [1, 43] verdankt sich genau diesem hohen Anspruch.

Um ihm gerecht zu werden, gaben die Entwickler der operationalen Diagnostik markante Rahmenbedingungen vor, die - mindestens in ihrer grundsätzlichen Orientierung - an das logisch-empiristische Wissenschaftsverständnis des Wiener Kreises um Rudolf Carnap (1891-1970) erinnern, ohne dass hier auf diesen für die Psychopathologie wesentlichen Kontext näher eingegangen werden kann [38].

Folgende, im Anschluss näher erläuterte Kernelemente prägen die operationalisierte psychiatrische Diagnostik:

- deskriptives Vorgehen,

- explizite Kriterien bzw. Kriterienverbindungen für jede Diagnose,

- Orientierung am Schweregrad,

- Mehrachsigkeit (mit Einschränkungen),

- Komorbiditätsprinzip,

- nominalistisches Verständnis von Diagnosen,

- ätiologische Neutralität (irreführend auch als „Theoriefreiheit" bezeichnet).

\section{Deskriptives Vorgehen}

Die Bezeichnung einer psychopathologischen Arbeitsweise als „deskriptiv“ betont den Anspruch, eine - im Idealfall direkt am Patienten beobachtbare - Symptomatik sei möglichst einfach, überprüfbar und übersichtlich gegliedert zu erfassen und zu benennen. In dieser Phase des diagnostischen Prozesses seien ätiologische oder pathogenetische Vorannahmen nicht von Belang.

„Deskription“ will gezielt die Assoziation des nüchternsachlichen Nachzeichnens von etwas „objektiv“ Vorhandenem hervorrufen, vergleichbar der passiven Funktion eines vom Benutzer gut justierten Fotoapparats. $\mathrm{Zu}$ bedenken ist aber, dass es sich bei jeder Beschreibung eines Verhaltens und Erlebens, gerade im Fall einer psychischen Störung, auch um eine Kommunikation zwischen den Beteiligten handelt. Zwar wird man ein Symptom wie „örtlich desorientiert“ präziser und „objektiver" feststellen können als Vorliegen und Ausprägungsgrad von „Gedankenausbreitung“. Dies ändert aber nichts an der grundsätzlichen Natur der psychopathologischen Deskription als eines Vorgangs, der nicht losgelöst vom Beziehungsaspekt der Akteure charakterisiert werden kann.

Das Beschreiben hängt nun einmal stark von der Art des Symptoms ab: Unmittelbar beobachtbares Verhaltens kann einfacher beschrieben werden als das subjektive Erleben der untersuchten Person. Jaspers hielt fest, „Seelisches“ zeige sich niemals unmittelbar, sondern stets vermittelt über Sprache, Schrift, Gestik, Mimik, künstlerischen Ausdruck, Verhalten. Umso wichtiger ist, sich bewusst zu machen, was eigentlich bei der deskriptiven Vorgehensweise „beschrieben" wird: Es sind dies v. a. das beobachtbare Verhalten einer Person sowie ihre Angaben über ihr aktuelles oder früheres Erleben. Jedoch fließen auch theoriegeleitete Annahmen des Untersuchers über das subjektive Erleben des Patienten ein, obwohl dieser selbst sich dazu u. U. ganz anders oder gar nicht äußert. Selbst Angaben Dritter (Fremdanamnese) können für die Art der „Deskription“ eines psychopathologisch auffälligen Verhaltens eine beträchtliche Rolle spielen.

Explizite Kriterien bzw. Kriterienverbindungen für jede Diagnose

Für jede Diagnose werden (mehr oder weniger präzise ausformulierte) explizite Kriterien und Kriterienverbindungen genannt. Die so entstehenden „Entscheidungsbäume“ generieren aber auch Zwänge: Sind die Kriterien einmal erfüllt, steht die Diagnose fest. Die klinisch durchaus sinnvolle Frage, ob aufgrund besonderer Umstände allenfalls eine andere (oder gar keine) Diagnose angemessen erscheint, beantwortet das operationalisierte Manual klar mit nein.

\section{Orientierung am Schweregrad (,quantitativer Zugang“)}

Operationalisierte Diagnosemanuale bevorzugen quantitative gegenüber qualitativen Aspekten psychopathologischer Phänomene. So wird etwa diagnostisch unterschieden zwischen einer leichten, mittelschweren und schweren depressiven Episode. Dies hat auch zur Folge, dass früher 
selbstverständliche, aber stark theoriegeleitete Unterscheidungen wie diejenige zwischen Neurose und Psychose oder, im Fall depressiver Störungen, zwischen endogenem und reaktivem Typ, in Misskredit gerieten und kaum noch Verwendung finden. Die ebenfalls qualitative Unterscheidung zwischen organisch begründbaren und nichtorganisch begründbaren psychischen Störungen wird allerdings nicht völlig abgeschafft, obwohl dies einem weiteren Grundprinzip, nämlich der Unabhängigkeit deskriptiver Diagnostik von ätiologischen Vorannahmen, widerspricht.

\section{Mehrachsigkeit}

Ein weiteres, ursprünglich stark betontes Element der operationalen Diagnostik konnte sich weder im ICD- noch im DSM-System wirklich durchsetzen, die Mehrachsigkeit (Multiaxialität). Um eine umfassende, nicht nur auf die klinische Symptomatik fokussierende Befunderhebung zu ermöglichen, sollte jede untersuchte Person auf verschiedenen „Achsen“ beurteilt werden. Beim DSM-IV waren dies 5 Achsen: Symptome im engeren Sinne, Persönlichkeitsmerkmale, körperliche Erkrankungen, psychosoziale Integration und Umfang psychischer Belastungen. In der praktischen Umsetzung der Multiaxialität war das DSM dem ICD stets um einiges voraus, doch hat jüngst das DSM-5 diesen Ansatz deutlich heruntergestuft vom integralen Bestandteil (wie noch im DSM-IV) auf die Option, v. a. Angaben zur Funktionalität im Alltag zusätzlich, aber eben nicht mehr auf einer eigenen (und obligatorischen) Achse zu dokumentieren.

\section{Konsequente Abbildung von Komorbiditäten}

Wer mit ICD-10 oder DSM-5 arbeitet, wird aufgefordert, beim selben Patienten alle Diagnosen zu stellen, deren Kriterien zum Untersuchungszeitpunkt erfüllt sind. Noch zu Zeiten des ICD-9 bestand ein deutlich höheres Risiko, nur die jeweils schwerste oder klinisch prominenteste Störung, etwa eine akute schizophrene Psychose, zu diagnostizieren und weitere behandlungsbedürftige Zustände zu übersehen, etwa eine Substanzabhängigkeit oder Persönlichkeitsstörung.

Nominalistisches (und nicht: realistisches) Verständnis psychiatrischer Diagnosen

Die operationalisierte Diagnostik stellt dem traditionellen, beispielhaft von der Kraepelinschen Psychiatrie vertretenen Streben nach Realdefinitionen die Beschränkung auf Nominaldefinitionen gegenüber: Bei Diagnosen nach ICD-10 und DSM-5 geht es gerade nicht um eine Aussage darüber, was Schizophrenie wirklich ist - das nämlich wäre eine Realdefi- nition -, sondern darum, klare Bedingungen festzulegen, bei deren zuverlässig festgestelltem Vorliegen von Schizophrenie gesprochen wird - eben der nominalistische Weg. In der Praxis kommt es gleichwohl mitunter zu einer unbemerkten „Reifizierung“ oder „Ontologisierung“, wenn nämlich der unkritische Anwender operational definierte Störungen für unverrückbare biologische (oder sonstige) Entitäten hält oder, wie Kraepelin es genannt hätte, für ,natürliche Krankheitseinheiten“. In diesem Fall würde er den Konstruktcharakter psychiatrischer Diagnosen verkennen.

Ätiologische Neutralität (,Theoriefreiheit“)

Im Idealfall sollen sich die kriteriengeleiteten Diagnosesysteme von ätiologischen Vorannahmen freihalten. Konkret heißt das, dass etwa mit der Diagnose einer ,schweren depressiven Episode ohne psychotische Merkmale" im Sinne der ICD-10-Kategorie F32.2 ausdrücklich keine Aussage zur Verursachung - neurobiologisch, psychodynamisch oder sozialpsychiatrisch - verknüpft wird. Diese Diagnostik versteht sich als unabhängig sowohl von der Ätiologiefrage als auch vom Bereich der Therapie, damit sie für Anwender unterschiedlichster theoretischer Provenienz nutzbar wird.

Oft wird in diesem Zusammenhang der irreführende Terminus „Theoriefreiheit“" verwendet. Natürlich kann damit nicht die Behauptung gemeint sein, operationalisierte Diagnosemanuale seien frei von theoretischen Voraussetzungen, denn das sind sie ohne Frage nicht. Vielmehr haben ICD-10 und DSM-5 den (hohen) Anspruch, eine Basis für die zuverlässige Beschreibung des klinisch Wahrnehmbaren darzustellen - und dies mit den geringst möglichen theoretischen „Importen“.

\section{Möglichkeiten und Grenzen der operationalen Diagnostik}

Die Entwicklung der operationalen Diagnostik seit den frühen 80er Jahren des 20. Jh.s wurde nicht nur wissenschaftlich, sondern auch in der Öffentlichkeit ebenso genau wie kritisch beobachtet [3, 10, 24, 25]. Dazu passt, dass das Erscheinen des DSM-5 nicht nur in seinem Herkunftsland, den USA, sondern auch in Europa eine ausgesprochen kontroverse öffentliche Diskussion hervorgerufen hat. Vor allem die massive Kritik von Frances [4], der an früheren Versionen des DSM federführend beteiligt war, schürte Sorgen wegen einer möglichen Inflation psychiatrischer Diagnosen bis hin zum Vorwurf der systematischen Pathologisierung der alltäglichen Lebenswelt. Da diese Debatte nicht selten polemische Züge annahm, scheint ein differenzierender Blick auf Möglichkeiten und Grenzen kriteriengeleiteter psychiatrischer Diagnostik angebracht. 
Wenn es um erreichte Verbesserungen geht, ist in erster Linie die deutlich höhere Reliabilität psychiatrischer Diagnosen zu nennen: Verschiedene Untersucher, vorausgesetzt, sie sind mit den Instrumenten hinreichend vertraut, gelangen mit einer deutlich höheren Wahrscheinlichkeit zum gleichen diagnostischen Ergebnis, als sie es ohne die kriteriengeleitete Diagnostik täten. Das Vorgehen nach dem Prinzip der Komorbidität verringert das Risiko, relevante Begleiterkrankungen und deren therapeutische Implikationen zu unterschätzen oder gar zu übersehen. Operationalisierte Manuale sind in der studentischen Ausbildung und der ärztlichen Weiterbildung wegen ihres hohen Strukturierungsgrads gut als inhaltliches „Gerüst“" zu verwenden. Der diagnostische Prozess und sein Ergebnis sind besonders dokumentations- und datenverarbeitungsfreundlich geworden, wobei allein dies natürlich noch keine qualitative Aufwertung bedeutet. Das System zwingt den Untersucher schließlich, sofern er es korrekt anwendet, sich mit jedem einzelnen Kriterium auseinanderzusetzen, was zu Sorgfalt und Vollständigkeit in der Befunderhebung beiträgt.

Operationalisiertes Diagnostizieren in der Psychiatrie stößt aber auch auf Schwierigkeiten und birgt Risiken: Wenn Deskription zu eng gefasst wird, etwa als eindeutiges Abbilden eines objektiven Tatbestands beim Patienten durch ein ebenso objektives Medium, den Untersucher, dann kommt es zu einem Überwiegen der formalen zuungunsten der inhaltlichen Aspekte. Die klinische Gesamtheit wird auf das vermeintlich „rein deskriptiv“ Erfassbare und qua Expertenkonsens Operationalisierbare reduziert. Dies führt fast zwangsläufig zu einer Vernachlässigung komplexer, nicht unmittelbar beobachtbarer, sich „nur“ im interpersonalen Kontakt erschließender Phänomene, wie etwa spezielle Anmutungsqualitäten im Kontakt mit psychotischen Menschen, biografisch verankerte psychische (Fehl-) Entwicklungen, charakteristische Züge der Persönlichkeit oder diagnostisch und therapeutisch relevante Aspekte der Arzt-Patient-Beziehung [21, 27, 32, 39].

Das Risiko einer radikalen Reduktion auf deskriptive Kriterien kann am Beispiel der Melancholiekonzeption Tellenbachs [40] veranschaulicht werden [35]. Deren tragende Begriffe, etwa die Inkludenz und Remanenz des Melancholikers, und ihre klinischen Erscheinungsformen finden, zumindest in vergleichbarer Differenziertheit, keinen Eingang in operationalisierte Diagnosemanuale. Das muss nicht zwingend ein Nachteil sein, sofern es nicht zur Folge hat, dass der gehaltvollen und therapierelevanten Analyse Tellenbachs die praktische Relevanz oder die Wissenschaftlichkeit abgesprochen werden, nur weil sie sich nicht in ICD-10 und DSM-5 widerspiegelt.

Einen nachhaltigen Versuch zur spezifischen Ergänzung der etablierten Diagnosemanuale stellt der Ansatz der „operationalen psychodynamischen Diagnostik“ (OPD) dar. Er akzeptiert im Grundsatz die kriterienorientierte Konzeption der Manuale, versucht aber, jenseits der deskriptiven Ebene psychodynamische und hermeneutische Elemente einzubeziehen [5, 37].

Eine unkritische „Reifizierung“ psychiatrischer Diagnose läge vor, verstünde man die operational definierten Einheiten als vom Untersucher völlig unabhängige und unveränderbare Krankheitseinheiten; ihr tatsächlicher Konstruktcharakter würde dann verkannt. Als verwandtes Risiko ist die Überschätzung des quantitativen zuungunsten des qualitativen Zugangs zu psychopathologischen Sachverhalten zu nennen. So etwa bestehen zwischen einer kurz dauernden und verständlichen, wenn auch ausgeprägten depressiven Reaktion im Rahmen eines Partnerkonflikts und einer „Melancholie“ im Sinne einer tiefgreifenden depressiv-psychotischen Verzerrung des Persönlichkeitsgefüges und Weltbezugs neben quantitativen auch qualitative Unterschiede. Beide Zustände könnten aber, sofern bei der „Melancholie“ keine klare Wahnsymptomatik vorliegt, nach ICD-10 als „schwere depressive Episode“ eingestuft werden. Die Diagnose allein würde dann die qualitativen Unterschiede jedenfalls nicht abbilden können.

$\mathrm{Zu}$ bedenken ist schließlich das Risiko, operationalisierte Diagnosesysteme nicht nur als nützliche psychiatrische Instrumente zu verstehen, sondern sie mit der Psychopathologie schlechthin gleichzusetzen. Diese würde dann reduziert auf die Lehre vom Umgang mit operationalen Kriterien unter Vernachlässigung aller kriteriologisch kaum oder gar nicht erfassbaren Phänomene. Schlimmstenfalls entstünde - und sei es noch so implizit - beim Anwender ein ,operationales Menschenbild“. Dies wäre sogar ein Schritt hinter die Assoziationspsychologie des frühen 19. Jh.s zurück, für die das psychische Feld einer Person die Summation von funktionierenden (oder im Krankheitsfall eben gestörten) Einzelleistungen war.

\section{Spezielle Aspekte der forensisch-psychiatrischen Situation}

Geht es in der operationalisierten Diagnostik darum festzustellen, ob eine bestimmte, durch Kriterien festgelegte Störung vorliegt oder nicht, verlangt das Gericht vom psychiatrischen Sachverständigen eine Äußerung über das Vorhandensein oder Fehlen von „Fähigkeiten“, im Fall des Strafrechts konkret der Fähigkeit, das Verbotene eines Handelns zu erkennen, sowie der Fähigkeit, das Handeln gemäß dieser Einsicht zu steuern. In erster Linie erwartet das Gericht also keine wissenschaftlich fundierten Antworten auf die Frage, ob ein Mensch sich in einer bestimmten (Tat-)Situation gesteuert hat oder nicht, sondern - erkenntnistheoretisch um einiges heikler - darauf, ob er zu dem gegebenen Zeitpunkt die genannten Fähigkeiten besessen hat oder eben nicht. 
Welche Rolle kann hier die operationalisierte Diagnostik spielen? Sie fußt, wie im klinischen Kontext erwähnt, wesentlich auf der aktuellen Verhaltensbeobachtung. In der forensischen Situation liegt der Fall aber anders, müssen hier doch häufig mehr oder weniger lange zurückliegende Verhaltensbeschreibungen beurteilt werden, zum einen aus erster Hand, als retrospektive Selbstschilderungen des Probanden, zum anderen von Dritten, also etwa dem Opfer, von Zeugen oder Bekannten. Möglichkeiten der Verzerrung ergeben sich dabei aus der unvermeidlichen und zudem noch stetigen Veränderungsprozessen unterworfenen subjektiven Einfärbung von Erinnerungen, von einer tendenziösen oder bewusst falschen Darstellung einmal ganz abgesehen.

Für die operationalisierte Diagnostik ist das Vorliegen bestimmter Kriterien logisch gleichwertig mit der Feststellung der betreffenden Diagnose. Der Schritt von den Kriterien zur Diagnose ist insoweit ein einfacher Algorithmus, er bedarf seitens des Untersuchers keines weiteren Bewertungsvorgangs. Anders in der Situation der Begutachtung: Aus dem retrospektiv festgestellten Vorliegen bestimmter beobachtbarer Verhaltensaspekte folgt mit Blick auf die Schuldfähigkeit noch nicht sehr viel. Unabdingbar ist es, zusätzliche psychopathologische, biografische, soziale und somatische Faktoren in einen Gesamtzusammenhang zu stellen. Beispielhaft deutlich wird dieser Kontext anhand der lang dauernden und kontroversen Diskussion um die Bedeutung von „Kriterienkatalogen“ bei der Beurteilung von affektiv akzentuierten Straftaten [8, 30, 33].

Nun ist heute die kriteriengeleitete Diagnostik in der forensischen Psychiatrie fast schon ein Standard, ja ihre Anwendung wird von manchen Gerichten als Conditio sine qua non eines wissenschaftlich begründeten psychiatrischen Gutachtens betrachtet [2]. Daher werden auch hier der Nutzen und die Limitationen der Methode behandelt.

Die im Vergleich zur voroperationalen Ära klarere Begrifflichkeit kann die Verständigung in foro zweifellos vereinfachen. Der Sachverständige hat die Möglichkeit, sich im Gespräch mit dem Juristen auf die Kriterienkataloge zu beziehen und diese am konkreten Fall zu erläutern. Orientiert sich der Sachverständige auch bei der mündlichen Erläuterung seines Gutachtens an den diagnostischen Richtlinien, so werden seine Entscheidungsprozesse transparenter. Erst recht gilt dies, wenn mehrere psychiatrische Sachverständige beauftragt sind, die zwar möglicherweise zu anders akzentuierten Ergebnissen gelangen können, aber sich idealiter auf denselben diagnostischen Kodex stützen.

Die Vergleichbarkeit psychiatrischer Diagnosen im Längsschnitt, in der Praxis nicht selten über viele Jahre, wird durch die konsequente Anwendung operationaler Kriterien deutlich verbessert. Dies führt nicht nur zu einer leichteren wissenschaftlichen Auswertbarkeit von Gutachten, sondern trägt auch zu einer stabileren Datenbasis für die Beurteilung von Langzeitverläufen bei, wie sie insbesondere bei Prognosegutachten anzustellen ist.

Interessant ist die Frage, ob die operationale Diagnostik für die Zuordnung psychiatrischer Sachverhalte zu juristischen Kategorien hilfreich sein kann, denn diese Zuordnung gehört ja zu den essenziellen Aufgaben eines psychiatrischen Sachverständigen. Die Antwort kann hier allerdings nur zwiespältig ausfallen: Zwar werden die relative Klarheit und die Präzision der operationalen Argumentation die Zuordnung der Diagnose etwa zu den vier psychopathologischen Merkmalen der Schuldfähigkeitsparagraphen, dem „ersten Stockwerk" bei der strafrechtlichen Begutachtung, transparenter und damit nachvollziehbarer machen. Jedoch nützt eine psychiatrische Diagnose allein - sei sie nach operationalen oder klinisch-intuitiven Grundsätzen erstellt - sehr wenig, wenn es um die von Gesetzes wegen zwingend erforderliche Quantifizierung des „ersten Stockwerks“ geht (,krankhaft", „tiefgreifend“, „schwer“) sowie um die komplexen Beurteilungsschritte des „Zweiten Stockwerks“ (erhebliche Beeinträchtigung oder Aufhebung der Einsichts- und/oder Steuerungsfähigkeit). Dabei ist es durchaus kein Widerspruch, dass auch die stark am Quantifizierungsgedanken orientierte operationale Diagnostik nicht alle Schweregradeinschätzungen abdeckt und abdecken kann, die im Rahmen des forensisch-psychiatrischen Entscheidungsfindungsprozesses erforderlich sind. Die jeweils benötigte Datenbasis ist unterschiedlich, was noch zur Sprache kommen wird.

Die Erkenntnis, dass aus einer psychiatrischen Diagnose, nach welcher Methodik auch immer sie erstellt worden sein mag, keine unmittelbaren Schlüsse auf rechtliche „Fähigkeiten“ gezogen werden dürfen, ist jüngeren Datums. Noch vor einigen Jahrzehnten pflegte man, genau umgekehrt, aus dem bloßen Vorliegen bestimmter Diagnosen, insbesondere solcher aus den psychotischen Formenkreisen, regelhaft direkt auf Schuldunfähigkeit oder Geschäftsunfähigkeit zu schließen. Janzarik [18] kritisierte dies als diagnostisches Vorurteil, wobei er seinerzeit nicht in erster Linie auf die operationale Diagnostik abzielte. Die Autoren der aktuellen Diagnosemanuale weisen denn auch explizit darauf hin, dass eine unbesehene Übertragung der für die klinisch-therapeutische Situation gedachten Algorithmen auf forensische Fragestellungen unzulässig ist und gravierende Fehlbeurteilung zur Folge haben kann. Plakativ gesagt: Operationale Diagnosen sagen per se (nahezu) nichts aus zur Ätiologie einer gegebenen Störung, zu ihrer Behandlung und zu ihren rechtlichen Konsequenzen.

Bei verkürzter Rezeption der Grenzen operationaler Diagnosemanuale mag der Jurist einen verzerrten Eindruck vom Prozess der psychiatrischen Diagnostik gewinnen: Er könnte diesen nämlich als Abbildung von quasi objektiven Symptomen missverstehen und so - dasselbe Problem wie im klinischen Bereich - die Bedeutung und die Komplexität der subtilen und biografisch orientierten psychopathologi- 
schen Befunderhebung unterschätzen. Aus psychiatrischer Perspektive bleibt diese eine Conditio sine qua non, unabhängig davon, ob anschließend eine operationale Diagnose gestellt wird oder nicht.

Jedem erfahrenen Gutachter und Juristen ist bekannt, wie wichtig die immer wieder auftretenden „Gestalten“ in einem Lebenslauf für das Verständnis eines individuellen Werdegangs und damit auch einer individuellen Tat sind. Mit der Metapher der „Gestalten“ sind hier z. B. wiederkehrende Konfliktmuster, die Qualität von Partnerbeziehungen und bevorzugte Problemlösungsstrategien des jeweiligen Individuums gemeint. Freilich darf man es der operationalen Diagnostik nicht unbesehen anlasten, dass sie für die Erfassung derartig komplexer Phänomene schlecht gerüstet ist, wurde sie doch dezidiert nicht zu diesem Zweck entwickelt.

Somit ergibt sich für die forensische Perspektive ein ähnliches Bild wie für die klinische: Die breite Anwendung einer operationalisierten psychiatrischen Diagnostik bringt klare Vorteile, kann aber ohne weitere Befunde und Informationen die Beantwortung der typischen gutachterlichen Fragen nach dem Vorliegen oder Fehlen einer bestimmten „Fähigkeit" keineswegs sicherstellen. An erster Stelle ist, wenn es um die Art der zusätzlich benötigten Informationen geht, der differenzierte psychopathologische Befund zu nennen, der auch komplexe, in den Kriterienkatalogen nicht oder zu knapp angeführte Sachverhalte erfasst. Eine solche differenzierte Befunderhebung soll sich aber keineswegs eilfertig den vorgegebenen juristischen Kategorien anpassen; sie bleibt auf genuin psychopathologischem Terrain. Sie wird dabei auch wahrnehmen, dass Einsicht und Steuerung zwar rechtlich getrennte, psychopathologisch aber sehr wohl - und zwar eng - verknüpfte Bereiche sind. Janzarik [18] führte in diesem Zusammenhang den für Juristen irritierenden Begriff der „Einsichtssteuerung“ ein.

Überdies erfordert die Biografie des Probanden eine umfassende forensische Würdigung, ebenso wie, darin eingebettet, seine spezielle Persönlichkeitsentwicklung in den verschiedenen Lebensphasen. Schließlich verlangt ein psychiatrisches Gutachten, insbesondere bei komplexer Fragestellung, die stetige Bezugnahme auf das klinischpsychopathologische Erfahrungswissen über psychische Störungen. Sass [34] hat dies mit dem praktisch relevanten Begriff des „psychopathologischen Referenzsystems“ umschrieben.

\section{Aktuelle Debatte}

Nach dieser Betrachtung der Möglichkeiten und Grenzen operationalisierter psychiatrischer Diagnostik für die Begutachtungssituation soll abschließend der Blick nochmals über die forensischen Fragen hinaus gerichtet werden. In den letzten Jahren entstand zur psychiatrischen Noso- logie generell und, als notwendige Folge, zur psychiatrischen Diagnostik ein breiter, ideenreicher Diskurs. Im Kern beschäftigt er sich (wieder einmal) mit der Reliabilitäts- und der Validitätsproblematik. Allerdings rückt nun die Validität stark in den Vordergrund, und zwar mit Blick auf das stark angewachsene Wissen über neurobiologische Zusammenhänge bei psychischen Störungen: Können sie allenfalls die zukünftige psychiatrische Diagnostik, etwa durch die systematische Einbeziehung von Endophänotypen und sonstigen Biomarkern, auf eine nachhaltigere Grundlage stellen? Diese vorwiegend in der klinischen Psychiatrie geführte Debatte wird ohne Frage bald auch das forensische Feld erfassen und die dort schon laufende Diskussion um die Aussagekraft psychopathologisch fundierter kriteriengeleiteter Diagnosen intensivieren.

Nun ist, daran soll ausdrücklich erinnert werden, das Problematisieren diagnostischer Konventionen in der Psychiatrie alles andere als neu. Methodenkritische Autoren stellten seit jeher die Fragen, ob Diagnosen wie Schizophrenie oder bipolare Erkrankung reale Krankheitseinheiten bezeichnen, ob sie Gruppen von Personen identifizieren, die mindestens ein notwendig gemeinsames, ,pathognomonisches“ Merkmal haben (etwa im Sinne des Begriffs „the core of schizophrenia“) oder ob sie lediglich Annäherungen und klinische Schätzungen auf der Grundlage von Symptom- und Syndromähnlichkeiten darstellen (können).

Spätestens seit den 80er Jahren des 20. Jh.s ist eine zunehmende Enttäuschung darüber zu spüren, dass es kaum gelang, stabil replizierbare neurowissenschaftliche Befunde entlang der konventionellen diagnostischen Trennlinien $\mathrm{zu}$ finden, also biologische Parameter, die klar differenziert hätten zwischen schizophrenem und bipolarem Formenkreis oder zwischen psychotischen und nichtpsychotischen Störungen. Nun begann die Karriere eines prägnant nosologiekritischen Begriffs: Denosologisierung [28, 41, 42]. Gemeint war (und ist) der Vorschlag, die psychiatrische Forschung nicht mehr an den klassischen diagnostischen Entitäten auszurichten, sondern an der nächst „tieferen“ Erkenntnisebene, also den klinischen Syndromen oder sogar den unmittelbar beobachtbaren Symptomen. Die pointierteste Kritik monierte, es sei gar nicht verwunderlich, dass zahlreiche neurowissenschaftliche Forschungsprojekte nicht zu replizierbaren, statistisch signifikanten Ergebnissen gelangten. Dies liege nicht an der neurowissenschaftlichen Methodik, sondern an den viel zu unpräzisen und $\mathrm{zu}$ wenig reliablen psychopathologischen Diagnosekriterien. Sie hätten fast zwangsläufig einen nichtakzeptablen Grad von Heterogenität in eigentlich auf Homogenität ihrer Mitglieder angewiesenen Forschungskohorten zur Folge. Genau deswegen müsse die psychiatrische Forschung sich von der Orientierung an (vermeintlichen) „Krankheiten“ entfernen, sie müsse eben „denosologisiert“ werden.

In dieselbe Richtung zielen die jüngst von Sartorius [29] gestellten Fragen: „Why do we need a diagnosis? Maybe 
a syndrome is enough?" Es ist kein Zufall, dass diagnosenkritische Ansätze zu einem Zeitpunkt weiteren Auftrieb erhielten, als sich die operationalisierte psychiatrische Diagnostik in Gestalt des vor zwei Jahren erschienenen DSM-5 markant und international einflussreich zu Wort meldete. Sartorius spielt mit seinen zugespitzten Fragen auf einen Gegenentwurf zum DSM-5 an, nämlich auf die jüngst viel diskutierten Research Domain Criteria (RDoC), die vom National Institute of Mental Health entwickelt wurden [15].

Die maßgeblichen Autoren im Umfeld der RDoC bevorzugen anstelle der vieldeutigen und daher zu allerlei Missverständnissen Anlass gebenden Bezeichnung ,personalisierte Medizin“ den Begriff „Präzisionsmedizin“. Diese komme, so Insel und Cuthbert [14], nunmehr auch in der Psychiatrie an. Gemeint ist der Anspruch, psychische Erkrankungen nicht mehr ausschließlich mithilfe mehr oder weniger reliabler, am Patienten direkt zu beobachtender klinischer Symptome zu diagnostizieren, sondern wesentlich direkter zu den als Grundlage vermuteten, besser quantifizierbaren neurobiologischen und neuropsychologischen Funktionsstörungen vorzudringen. Die Autoren plädieren dafür, die von ihnen als fruchtlos bezeichnete Debatte um das Leib-Seele-Problem zu beenden; sie habe ein vertieftes Verständnis psychischer Störungen mehr behindert als gefördert. Vielmehr seien die Syndrome, die traditionell als psychische Störungen klassifiziert würden, zukünftig vor dem Hintergrund eines Verständnisses des Gehirns als lernendem und sich stets neu organisierendem System zu konzeptualisieren.

Das von den Autoren der RDoC skizzierte optimistische Forschungsszenario wird auch für forensische Fragestellungen Bedeutung erlangen: Ziel sei es, die Ausrichtung der Forschung von den ICD-10- bzw. DSM-5-Diagnosen zu lösen. Vielmehr seien große Patientengruppen - etwa solche mit Auffälligkeiten der Affektivität im weitesten Sinne - bezüglich verschiedener Domänen zu untersuchen. Dabei stehen aktuell die folgenden Bereiche im Mittelpunkt des Interesses:

- genetische Ebene (polygener Risiko-Score),

- neurophysiologische Ebene (neuronale Erregungsmuster, z. B. im insulären Kortex),

- physiologische Ebene (z. B. Entzündungsparameter),

- neuropsychologische und Verhaltensebene (z. B. reduzierte affektive Schwingungsfähigkeit beim Depressiven),

- „life events“(z. B. interaktionale und kulturelle Faktoren).

Das erklärte Ziel ist, durch die Erfassung von Daten auf allen diesen Ebenen Gruppen von Personen zu identifizieren, die sich durch empirisch replizierbare Auffälligkeiten in den genannten Domänen auszeichnen. Ob diese Gruppen den heutigen ICD-10- oder DSM-5-Kriterien für bestimmte psychiatrische Diagnosen entsprechen werden, ist nicht von Relevanz; vermutet wird, dass die Übereinstimmung eher gering sein wird. Wohl aber - aus Sicht der Befürworter der entscheidende Vorteil dieses Ansatzes - könnten mit den derart gewonnenen homogenen Clustern von Personen Replikations- und Therapiestudien durchgeführt werden. So werde man dem Idealbild einer „Präzisionsmedizin“ weit näher kommen, als dies heute der Fall sei.

Mit Blick auf die Schizophrenieforschung hat Pratt den programmatischen Kern des Ansatzes sehr pointiert formuliert:

Ein entscheidendes Ziel der Schizophrenieforschung besteht darin, diese Störung auf biologischer Ebene zu erfassen und nicht über ihr klinisches Erscheinungsbild. ([26], übersetzt von P.H.)

Hier ist nun ein Caveat angebracht. Die zitierte Kernaussage macht eben nicht nur einen methodischen Vorschlag zur psychiatrischen Forschung, sondern sie nimmt auch eine starke Wertung vor, freilich eine kaum hinterfragte: Wenn es wesentliches Ziel der Forschung ist, die klinisch-psychopathologische Erfassung durch neurobiologische Daten und Begriffe zu ersetzen (notabene: zu ersetzen, nicht etwa zu ergänzen!), dann wird die psychopathologische Ebene notwendig als wissenschaftlich zweitrangig, wenn nicht sogar irrelevant verstanden. Hier aber entsteht ein Problem: Originelle, eingetretene Pfade verlassende neurowissenschaftliche Forschungsstrategien sind das eine. Die reflexartig mitvollzogene pauschale Diskreditierung anderer Perspektiven, etwa der deskriptiv-psychopathologischen, der phänomenologischen und der hermeneutischen, ist das andere.

Bezogen auf den Anspruch der RDoC ist daher festzuhalten, dass tradierte diagnostische Begriffe wie derjenige der Schizophrenie sehr wohl durch modernere Konzepte ersetzt werden können (und sollen), sofern dafür überzeugende wissenschaftliche Argumente sprechen. Dies darf aber nicht dazu führen, dass die mit dem kritisierten traditionellen Begriff verknüpfte reiche Denktradition unbesehen - gleichsam nur deswegen, weil sie eine Tradition ist - mitabgeschafft wird.

Jede Weiterentwicklung der psychiatrischen Diagnostik und Nosologie sollte dazu beitragen, die unproduktive bloße Gegnerschaft von psychopathologischen und neurowissenschaftlichen Zugangswegen zu verlassen. Beide sollten sich wechselseitig als autonome, aber aufeinander angewiesene Felder respektieren. Der Ansatz der RDoC ist originell und zukunftsweisend. Dies gilt auch für die forensische Psychiatrie. Man denke nur an so komplexe Forschungsbereiche wie Aggressivität und Dissozialität oder an die methodische Herausforderung der Prognose delinquenten Verhaltens. Allerdings darf bei der Weiterentwicklung der RDoC der in der psychiatrischen Ideengeschichte so häufig anzutreffende Fehler nicht wiederholt werden, die je eigene Position plakativ oder still zu dogmatisieren und damit vermeintlich gegen Kritik zu immunisieren. 


\section{Resümee}

Die folgenden, zugespitzt formulierten Fragen und Antworten bringen die Kerngedanken der Arbeit zum Ausdruck.

I. Brauchen wir überhaupt (noch) eine systematische psychiatrische Klassifikation und Diagnostik, die über die Erfassung individueller Symptomkonstellationen hinausgeht?

Ja. Die Vorteile einer sorgfältig angewandten Klassifikation und Diagnostik überwiegen klar die Risiken, sofern alle wissenschaftlichen Erkenntnisebenen konsequent einbezogen werden. Dies gilt in gleicher Weise für den klinischen, wissenschaftlichen und forensischpsychiatrischen Bereich.

II. Sind psychiatrische Klassifikation und Diagnostik das Ergebnis objektiver Messungen oder subjektiver Eindrücke?

Weder das eine noch das andere. Es handelt sich um wissenschaftliche Konventionen, die aber nicht beliebig sind, sondern sich auf überprüfbare psychopathologische, somatische und Verlaufskriterien sowie auf benennbare (und damit kritisierbare) theoretische Vorannahmen stützen.

Mit Blick auf die aktuelle Debatte um die forensische Bedeutung von quantifizierbaren neurobiologischen Parametern („Biomarkern“) folgt daraus, dass allein aus der Absenz eines objektiven (etwa neurophysiologischen oder bildgebenden) Untersuchungsbefunds nicht geschlossen werden darf, die betreffende Person habe keine straf-, zivil- oder sozialversicherungsrechtlich relevante psychische Störung. Aber, allen wohlfeilen Vereinfachungen zum Trotz, es gilt auch die umgekehrte Relation: Ein eindeutiger objektiver Befund, etwa eine magnetresonanztomographisch nachgewiesene frontale Hirnatrophie, erlaubt keinen unmittelbaren Rückschluss auf eine zu Leidensdruck und forensisch relevanter Leistungsbeeinträchtigung führende psychische Störung.

III. Erlaubt eine psychiatrische Diagnose eo ipso Rückschlüsse in juristischem Kontext?

Nein. Es braucht zwingend zusätzliche Informationen v. a. zu den individuellen Ressourcen und psychosozialen Kompetenzen (,Funktionalität im Alltag“). Dies gilt für alle psychiatrischen Diagnoseverfahren, also auch für die operationalisierte Diagnostik nach ICD-10 und DSM-5.
Selbstverständlich, so das abschließende Fazit, sind das DSM-5 und die in absehbarer Zeit zu erwartende ICD-11 für die forensische Psychiatrie von Bedeutung. Jedoch sollte diese nicht überschätzt werden. Auch darf die seit 2013 beobachtete plakative Aufregung über potenziell negative Effekte des DSM-5 den nüchternen Blick auf dieses Manual und seine Auswirkungen auf die psychiatrische Begutachtung nicht trüben. Das tatsächliche Risiko des DSM-5 (und wohl auch dasjenige der zukünftigen ICD-11) liegt nicht in einer unkontrollierten Ausweitung von ,forensisch relevanten“ psychiatrischen Diagnosen. Solche gibt es nämlich gar nicht: Diagnosen, welcher Provenienz auch immer, führen nie $\mathrm{zu}$ einer unmittelbaren forensisch-psychiatrischen Konsequenz.

Durchaus schwer wiegt aber das Risiko, durch eine klischeehafte Debatte um die psychiatrische Diagnostik eine in der Öffentlichkeit tendenziell stets bereitliegende negative Konnotation der Psychiatrie zu verschärfen - als eines Fachs nämlich, das sich durch die Hintertür der seit 1980 markant erhöhten Zahl von operational definierten Diagnosen in unredlicher Weise in das Verhalten und Erleben ,normaler", sprich psychisch gesunder Personen einmische.

Die zukünftige Diskussion um die forensisch-psychiatrische Diagnostik darf nicht zu stark auf formale Details von ICD-10 und DSM-5 fokussieren. Denn bei aller Kritikwürdigkeit in einzelnen Bereichen sind es mit Sicherheit nicht ICD-10 und DSM-5 als solche, die eine Gefahr für Qualität und Glaubwürdigkeit der psychiatrischen Begutachtung darstellen. Die eigentlichen Risiken resultieren vielmehr aus einer mangelnden psychopathologischen Sorgfalt bei der Befunderhebung, der Vernachlässigung der Diagnostikforschung zu gutachterlich relevanten Störungen sowie der Unterschätzung des Werts, der in diesem komplexen Umfeld einem ernsthaften und kontinuierlichen interdisziplinären Dialog zwischen Psychiatrie und Jurisprudenz zukommt.

\section{Einhaltung ethischer Richtlinien}

Interessenkonflikt P. Hoff gibt an, dass kein Interessenkonflikt vorliegt.

Dieser Beitrag beinhaltet keine Studien an Menschen oder Tieren.

\section{Literatur}

1. APA (American Psychiatric Association) (2013) Diagnostic and statistical manual of mental disorders, Fifth Edition (DSM-5). APA, Arlington

2. Boetticher A, Nedopil N, Bosinski HA, Sass H (2005) Mindestanforderungen für Schuldfähigkeitsgutachten. Nervenarzt 76:1154-1160

3. Craddock N, Mynors-Wallis L (2014) Psychiatric diagnosis: impersonal, imperfect and important (editorial). Br J Psychiatry 204:93-95 
4. Frances A (2013) Normal: Gegen die Inflation psychiatrischer Diagnosen. Dumont, Köln

5. Freyberger HJ, Dierse B, Schneider W et al (1996) Operationale psychodynamische Diagnostik (OPD) in der Erprobung. Ergebnisse einer multizentrischen Anwendungs- und Praktikabilitätsstudie. Psychother Psychosom Med Psychol 46:356-366

6. Hoff P (1994) Emil Kraepelin und die Psychiatrie als klinische Wissenschaft. Ein Beitrag zum Selbstverständnis psychiatrischer Forschung. Springer, Berlin

7. Hoff P (2007) Arthur Kronfeld (1886-1941): Ein vergessener, aber überaus aktueller psychopathologischer Denker. Sozialpsychiatrische Informationen 37:15-17

8. Hoff P (2010) Vorgestalten und Selbstkorrumpierung auf dem Weg zum Affektdelikt. Forensische Psychiatrie, Psychologie. Kriminologie 4:240-247

9. Hoff P (2012) Arthur Kronfeld (1886-1941) und das Postulat einer „autologischen Psychiatrie“. In: Holdorff B, Kumbier E (Hrsg) Schriftenreihe der Deutschen Gesellschaft für Geschichte der Nervenheilkunde (DGGN), Bd 18. Königshausen \& Neumann, Würzburg, S 233-250

10. Hoff P (2014) Psychiatrische Krankheitsbilder, mit einem Ausblick auf DSM-5. In: Riemer-Kafka G (Hrsg) Psyche und Sozialversicherung. Luzerner Beiträge zur Rechtswissenschaft. Bd 81 . Schulthess, Zürich, S. 1-26

11. Hoff P (2015) The Kraepelinian Tradition. Dialogues Clin Neurosci $17: 31-41$

12. Hoff P, Sass H (2010) Psychopathologische Grundlagen der forensischen Psychiatrie. In: Kröber HL, Dölling D, Leygraf N, Sass H (Hrsg) Handbuch der Forensischen Psychiatrie. Band 2: Psychopathologische Grundlagen und Praxis der Forensischen Psychiatrie im Strafrecht. Springer, Berlin, S 1-156

13. Huber G (1997) Kurt Schneider (1887-1967) - ein Psychiater in seiner Zeit. Spektrum Psychiatr Psychother Nervenheilk 26:102-106

14. Insel TR, Cuthbert BN (2015) Brain disorders? Precisely. Precision medicine comes to psychiatry. Science 348:499-500

15. Insel TR, Cuthbert BN, Garvey M, Heinssen R, Pine DS, Quinn K, Sanislow C, Wang P (2010) Research domain criteria (RDoC): toward a new classification framework for research on mental disorders. Am J Psychiatry 167:748-751

16. Janzarik W (1979) Psychopathologie als Grundlagenwissenschaft. Enke, Stuttgart

17. Janzarik W (1984) Jaspers, Kurt Schneider und die Heidelberger Psychopathologie. Nervenarzt 55:18-24

18. Janzarik W (1995) Grundlagen der Schuldfähigkeitsüberprüfung. Enke, Stuttgart

19. Jaspers K (1912) Die phänomenologische Forschungsrichtung in der Psychopathologie. Zschr ges Neurol Psychiat 9:391-408

20. Jaspers K (1913) Allgemeine Psychopathologie. Springer, Berlin; (1946) 4., völlig neu bearbeitete Auflage. Springer, Berlin

21. Kraus A (1991) Phänomenologische und symptomatologisch-kriteriologische Diagnostik. Fundamenta Psychiatrica 5:102-109

22. Kronfeld A (1920) Das Wesen der psychiatrischen Erkenntnis. Springer, Berlin

23. Maatz A, Hoff P, Angst J (2015) Eugen Bleuler's schizophrenia - a modern perspective. Dialogues Clin Neurosci 17:43-49

24. Maj M (2011) Psychiatric diagnosis: pros and cons of prototypes vs. operational criteria (editorial). World Psychiatry 10:81-82
25. Paris J, Phillips J (Hrsg) (2013) Making the DSM-5. Concepts and controversies. Springer, London

26. Pratt J (2015) Schizophrenia in the 21 st century: new insights and translation into improved therapies (editorial). J Psychopharm 29:83-84

27. Priebe S (1989) Über die Subjektivität der psychiatrischen Diagnose. Psychiatr Prax 16:86-89

28. Read J, Mosher LR, Bentall RP (Hrsg) (2004) Models of Madness. Psychological, social and biological approaches to schizophrenia. Brunner-Routledge, New York

29. Sartorius N (2015) Why do we need a diagnosis? Maybe a syndrome is enough? (Guest editorial). Dialogues Clin Neurosci 17:6-7

30. Sass H (1983) Affektdelikte. Nervenarzt 54:557-572

31. Sass H (1987) Die Krise der psychiatrischen Diagnostik. Fortschr Neurol Psychiatr 55:355-360

32. Sass H (1990) Operationalisierte Diagnostik in der Psychiatrie. Nervenarzt 61:255-258

33. Sass H (1993) Affektdelikte - Interdisziplinäre Beiträge zur Beurteilung von affektiv akzentuierten Straftaten. Springer, Berlin

34. Sass H (2008) Psychische Störungen und Schuldfähigkeit - Ein psychopathologisches Referenzsystem. Die Psychiatrie 5:182-189

35. Schmidt-Degenhard M (1985) Zum Melancholiebegriff J C A Heinroths. In: Nissen G, Keil G (Hrsg) Psychiatrie auf dem Wege zur Wissenschaft. Thieme, Stuttgart, S 12-18

36. Schneider K (1992) Klinische Psychopathologie, 15. Aufl. Thieme, Stuttgart

37. Schneider W, Klauer T, Freyberger HJ (2008) Operationalized psychodynamic diagnosis in planning and evaluating the psychotherapeutic process. Eur Arch Psychiatry Clin Neurosci 258(Suppl. 5):86-91

38. Schwartz MA, Wiggins OP (1986) Logical empiricism and psychiatric classification. Compr Psychiatry 27:101-114

39. Schwartz MA, Wiggins OP, Norko MA (1989) Prototypes, ideal types, and personality disorders: the return to classical psychiatry. J Personal Disord 3:1-9

40. Tellenbach H (1983) Melancholie, 4. Aufl. Springer, Berlin Heidelberg New York

41. Van Os J, Tamminga C (2007) Deconstructing psychosis. Schizophr Bull 33:861-862

42. Van Praag HM, Kahn RS, Asnis GM, Wetzler S, Brown SL, Bleich A, Korn ML (1987) Denosologization of biological psychiatry or the specificity of 5-HT disturbances in psychiatric disorders. J Affect Disord:173-193

43. WHO (World Health Organization) (1992) The ICD-10 classification of mental and behavioural disorders. Clinical descriptions and diagnostic guidelines. WHO, Geneva. [deutsch: Weltgesundheitsorganisation (2000) Internationale Klassifikation psychischer Störungen: ICD-10 Kapitel V (F). Klinisch-diagnostische Leitlinien. Übersetzt und herausgegeben von H. Dilling, W. Mombour, M. H. Schmid unter Mitarbeit von E. Schulte-Markwort. 4. durchgesehene und ergänzte Auflage. Hans Huber, Bern Göttingen Toronto Seattle]

44. Zachar P (2000) Psychiatric disorders are not natural kinds. Philos Psychiatr Psychol 7:167-182

45. Zachar P, Kendler KS (2007) Psychiatric disorders: a conceptual taxonomy. Am J Psychiatry 164:557-565 